\title{
Reform des Beschäftigtendatenschutzes
}

Das Recht auf informationelle Selbstbestimmung (Art. 2 Abs. 1 i. V. m. Art. 1 Abs. $1 \mathrm{GG}$ ) gilt auch im Rahmen eines Arbeitsverhältnisses. Dessen ungeachtet scheint die Achtung der Privatsphäre des Beschäftigten am Arbeitsplatz mehr eine Wunschvorstellung zu sein, werden die Schlagzeilen der letzten Monate zu Arbeitnehmerscreenings und Datenmissbräuchen in größeren Unternehmen (u.a. Deutsche Bahn, Lidl) betrachtet. Es ist daher wenig überraschend, dass das Thema "Beschäftigtendatenschutz" und dessen gesetzliche Ausgestaltung inzwischen sowohl in der Öffentlichkeit als auch in der Politik immer mehr Relevanz erhält und Gegenstand heftiger Diskussionen ist. Schon vor Bekanntwerden der zahlreichen Datenskandale wurde die Notwendigkeit eines eigenen Beschäftigtendatenschutzes in der Literatur jahrelang diskutiert, von der Politik gefordert und sogar im Vertrag zur Herstellung der Einheit Deutschlands vereinbart, bis dann mit der Einführung des § 32 BDSG durch die Novelle II des Bundesdatenschutzgesetzes zum 1.9.2009 wegen des nun vorgelegten umfassenderen Entwurfs eines Beschäftigtendatenschutzes zumindest eine vorläufige Regelung des Beschäftigtendatenschutzes gefunden schien. Die seit 2010 bestehende Regelung führt aber wegen der vielen ungeklärten Auslegungsfragen zu zahlreichen Auseinandersetzungen über die Anwendbarkeit des §32 BDSG und die erlaubte Verarbeitung von Beschäftigtendaten in den Unternehmen. Es ist daher wenig erstaunlich, dass es nicht einmal ein Jahr dauerte, bis das Bundesministerium des Innern einen Referentenentwurf zum Beschäftigtendatenschutz vorlegte, dem dann der Gesetzentwurf zur Regelung des Beschäftigtendatenschutzes der Bundesregierung folgte. Zu diesem Entwurf hat der Bundesrat in seiner Sitzung am 5.11.2010 eine Stellungnahme beschlossen (BR-Drucksache 535/10 vom 5.11.2010), mit der zahlreiche formale und inhaltliche Änderungen gefordert werden. Die Gegenäußerung der Bundesregierung steht noch aus.

Sowohl der Entwurf selbst als auch die Änderungswünsche des Bundesrates sind jedoch schon jetzt zahlreicher Kritik ausgesetzt. Die Beiträge dieses Heftes zeigen die zu erwartenden Auswirkungen der geplanten Änderungen auf und tragen zur gegenwärtigen Diskussion bei. Zu Anfang beschäftigen sich die Autoren Martin Sebastian Haase, Thorsten Heermann und Wolfgang Rottwinkel in ihrem Beitrag mit den durch die geplante Reform des Beschäftigtendatenschutzes zu erwartenden Neuerungen im Bewerbungs- und Einstellungsverfahren. Im Anschluss werden im Aufsatz von Bernd Schmidt und Christian Jakob die Auswirkungen der Reform auf die Zulässigkeit IT-gestützter Compliance- und Risikomanagementsysteme dargestellt, bevor Christoph Bausewein die Reichweite des $\S 32$ Abs. 2 BDSG und die durch den Gesetzentwurf zu erwartenden Neuerungen untersucht. Die Änderungen der datenschutzrechtlichen Anforderungen an eine Videoüberwachung durch die geplante Reform des Beschäftigtendatenschutzrechts und deren Auswirkung auf die Praxis, wird von Bernd Seifert näher beleuchtet. Im Forum setzt sich dann Karin Schuler kritisch mit der Stellungnahme des Bundesrates zum Gesetzentwurf der Bundesregierung zur Regelung des Beschäftigtendatenschutzes auseinander. Schließlich erläutert Edgar Rose die Bedeutung einer Betriebsvereinbarung im Rahmen von Datenschutzregelungen im Beschäftigtenverhältnis.

Britta Alexandra Mester 